\title{
The Tsc1-Tsc2 complex influences neuronal polarity by modulating TORC1 activity and SAD levels
}

\author{
Jill Wildonger, Lily Yeh Jan, and Yuh Nung Jan ${ }^{1}$ \\ Department of Physiology and Department of Biochemistry, Howard Hughes Medical Institute, University of California \\ at San Francisco, San Francisco, California 94143, USA
}

Neuronal function depends on the specification of neuronal processes as axons or dendrites. In this issue of Genes \& Development Choi and colleagues (pp. 24852495) show that without Tuberous Sclerosis Complex 1 (Tsc1) or Tsc2, molecules linked to the autosomal dominant disease tuberous sclerosis, an increase in the activity of the translational regulator Target of Rapamycin 1 (TORC1) causes neurons to have multiple axons and the translation of SAD kinase increases as well. Thus, in addition to the kinase LKB1, the Tsc1-Tsc2 complex, acting through TORC1, also modulates SAD to regulate axon formation.

The majority of mammalian neurons are polarized, meaning that they have specialized processes that either receive signals (dendrites) or send signals (axons). Neurons typically have multiple dendrites but only a single axon, and a neuron's polarity ensures that information flows directionally within a neuronal circuit. Recent studies have identified several molecules, including the kinases SAD and LKB1 (Par 4), as regulators of neuronal polarity in the developing murine brain (Kishi et al. 2005; Barnes et al. 2007; Shelly et al. 2007). Although SAD has a necessary role in creating neuronal polarity, SAD kinases are not found specifically in one compartment, but localize to both axons and dendrites (Kishi et al. 2005). Recent work revealed that LKB1 activates SAD preferentially within axons (Barnes et al. 2007), indicating that $\mathrm{SAD}$ is differentially active in axons versus dendrites. Now, Choi et al. (2008) present evidence that the translation of SAD is also regulated (Fig. 1). Choi et al. (2008) found that the Tsc1-Tsc2 complex restricts axon formation by inhibiting the translational regulator TORC1. In wild-type neurons Akt inactivates the Tsc1-Tsc2 complex in nascent axons, and in neurons with reduced

[Keywords: Neuronal polarity; tuberous sclerosis complex, TSC; SAD kinase; autism]

${ }^{1}$ Corresponding author.

E-MAIL yuhnung.jan@ucsf.edu; FAX (415) 476-5774.

Article is online at http://www.genesdev.org/cgi/doi/10.1101/gad.1724108.
Tsc1-Tsc2 complex activity multiple axons form. Western blot analysis revealed that the Tsc1-Tsc2 complex, acting through TORC1, modulates the amount of SAD protein within neurons. As TORC1 has been previously implicated in local protein synthesis, these findings suggest a model in which inhibiting Tsc1-Tsc 2 complex activity within a neuronal process leads to increased TORC1 activity and elevated amounts of SAD protein, which in turn promotes axon formation.

The Tsc1-Tsc2 complex: its role in human disease and cell growth

Tuberous Sclerosis Complex (TSC) is an autosomal dominant human disease characterized by the appearance of benign tumors, called hamartomas, in multiple organs and tissues, most commonly the skin, kidney, heart, and nervous system (Crino et al. 2006; Huang and Manning 2008). Mutations in two conserved genes, TSC1 and TSC2, underlie the majority of TSC cases. Neurological deficits associated with TSC include epilepsy, mental retardation, and autism. In the brains of TSC patients there are typically small regions where the cortical layers are no longer clearly defined. Referred to as cortical tubers, these areas also contain abnormally shaped neurons, large astrocytes, and cells of an unclear origin called "giant cells," which have unusual processes that resemble neither axons nor dendrites (Crino 2004). Cortical tubers likely result from perturbations in normal neuronal development, although it is not known what mechanism(s) contribute to cortical tuber formation.

Tsc1 and Tsc2, which are also known as hamartin and tuberin, respectively, interact with each other to form a complex. Tsc2 has a C-terminal GTPase-activating domain whose main target is the GTPase Rheb. Tsc1, which does not have any recognizable functional domains, likely prevents the degradation of Tsc2. The formation of tumors in TSC patients suggested a role for the Tsc1-Tsc2 complex in regulating cell proliferation and growth, and significant evidence supporting this idea initially came from three separate studies carried out in the fruit fly (Gao and Pan 2001; Potter et al. 2001; Tapon et al. 2001). Loss of either Tsc1 or Tsc2 resulted in tissue 


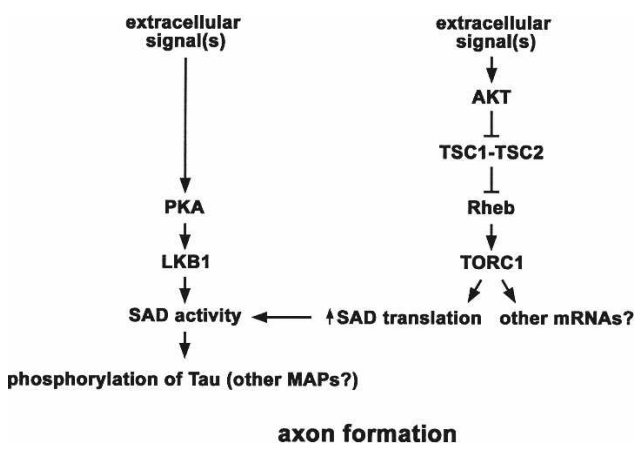

Figure 1. Two pathways that promote axon formation by regulating SAD activity or SAD protein levels. Prior studies revealed that $\mathrm{SAD}$ activity is positively regulated by LKB1, which is activated in response to extracellular signals such as BDNF (Barnes et al. 2007; Shelly et al. 2007). In a new report published in this issue of Genes \& Development, Choi et al. (2008) present evidence for a parallel pathway, regulated by the Tsc1-Tsc2 complex and TORC1, which modulates SAD protein levels. This new work suggests that local protein synthesis in the nascent axon is critical for neurons to polarize.

overgrowths characterized by an increase in both cell number and cell size. Similar defects in cell proliferation and growth had been previously observed by perturbing insulin receptor signaling, and genetic epistasis experiments placed Tsc1 and Tsc2 downstream from PI3K and Akt and upstream of the translational regulator ribosomal S6 kinase (S6K). These studies provided the first in vivo evidence that Tsc1 and Tsc2 are part of a conserved signaling pathway that has a crucial function in regulating tissue growth.

A key regulator of cell growth in many different organisms is Target of Rapamycin (Tor), which can function in two functionally distinct heteromeric complexes, Tor complex 1 (TORC1) and Tor complex 2 (TORC2) (Swiech et al. 2008). While the cellular function of TORC2, which is rapamycin insensitive, is still being elucidated, the more widely studied rapamycin-sensitive TORC1 has roles in multiple cellular processes. The binding of a growth or trophic factor to its receptor initiates a cascade of phosphorylation events, briefly summarized as follows: PI3K phosphorylates and activates Akt, which in turn phosphorylates Tsc2, inactivating the Tsc1-Tsc2 complex. In the absence of Tsc1-Tsc2 complex activity, the GTPase Rheb binds GTP, which leads to the activation of TORC1. TORC1 positively regulates the activity of S6K and other translational regulators that promote cell growth. The loss of Tsc1-Tsc2 function results in an increase in TORC1 activity, and tissue from TSC patients show elevated levels of phosphorylated S6K (Crino et al. 2006). Support for the pathological significance of increased TORC1 activity in TSC came with the findings that inhibiting TORC1 activity in mouse models of TSC reversed some of the phenotypes, such as tumor growth, associated with TSC. Encouragingly, recent data indicates that rapamycin can lessen the severity of neurological deficits present in Tsc1 or Tsc2 mutant mice (Ehninger et al. 2008; Meikle et al. 2008).
However, treating postnatal mice with rapamycin does not reverse the mild cortical dysplasia phenotype, suggesting that defects in cortical architecture result from the loss of Tsc1-Tsc2 complex activity earlier during development (Meikle et al. 2008).

\section{The Tsc1-Tsc2 complex acts upstream of TORC1 in the dendrites of mature neurons}

Given the cortical defects and cognitive deficits associated with TSC, it is important to know more about the function of Tsc1 and Tsc2 in the nervous system. Several approaches have been taken including creating different strains of Tsc1 and Tsc2 mutant mice that exhibit many of the neurological features of TSC, including cortical lesions, epilepsy, and learning deficits (Uhlmann et al. 2002; Meikle et al. 2007, 2008; Ehninger et al. 2008). As in other tissues, the loss of Tsc1-Tsc2 complex function in neurons results in an increase in phosphorylated S6K, indicating that Tsc1 and Tsc2 also modulate TORC1 activity in the nervous system (Tavazoie et al. 2005). Morphologically, neurons lacking Tsc1 or Tsc2 have enlarged somas and the density of spines along the mutant dendrites is significantly reduced (Tavazoie et al. 2005; Meikle et al. 2008). Dendritic spines, as their name suggests, are small protrusions along the dendritic shaft that are points of excitatory synaptic contact with other neurons. Changes in spine density and morphology correlate with changes in neuronal activity; moreover, abnormal spine shape and number are associated with cognitive and behavioral deficits in mice and humans (Halpain et al. 2005). Results from hippocampal slice cultures indicate that reducing Tsc1 or Tsc2 function increases spine length and spine head size, but these phenotypes were not seen in the brains of Tsc1 conditional knockout mice. The TORC1 inhibitor rapamycin suppressed the increase in soma size and modestly increased spine density in Tsc1 and Tsc2 mutant neurons, indicating that TORC1 acts downstream from Tsc1 and Tsc2 (Tavazoie et al. 2005; Meikle et al. 2008). TORC1 has been previously shown to regulate dendrite and dendritic spine growth by promoting protein synthesis locally (Jaworski and Sheng 2006). Although constitutively active Akt (Myr-Akt) decreases spine density, similar to the loss of Tsc2, a Tsc2 mutant that cannot be phosphorylated by Akt surprisingly does not suppress the Myr-Akt phenotype (Tavazoie et al. 2005). While these results suggest that Tsc1 and Tsc2 regulate dendrite spine density by modulating TORC1 activity, the relationship between Akt and the Tsc1-Tsc2 complex in this context is unclear.

The work described above reveal that the Tsc1-Tsc2 complex is necessary in mature neurons for normal dendrite morphology and function. These studies, however, did not directly address the question of what role the Tsc1-Tsc2 complex has during earlier stages of neuronal development. This question is of particular interest, given that the cortical tubers likely originate from defects early in neuronal development. 


\section{Establishing polarity in neurons}

The process of neuronal polarization has been primarily studied in vitro by observing how the axons and dendrites of cultured hippocampal neurons form. These studies have yielded insight into cytoskeletal changes that occur during neuronal polarization. They also uncovered intrinsic signaling pathways that regulate axon versus dendrite formation. Whether and how these events and pathways might contribute to neuronal polarization in vivo have only begun to be elucidated. As mentioned above, an in vivo role in neuronal polarization has been established for SAD kinases and LKB1 in mammals, and now the Tsc1-Tsc2 complex can be added to this list.

There are similarities and differences in how neurons polarize in culture versus in the developing nervous system. The polarization of hippocampal neurons in culture has been divided into five stages, starting with neurons first forming lamellepodia (stage 1) and then extending multiple processes of equivalent length (stage 2). During the transition from stage 2 to stage 3 , one neurite elongates more rapidly than the others and becomes the nascent axon (Dotti et al. 1988). The formation of the axon is the first sign of polarization, and thus most studies have focused on the mechanisms underlying axonogenesis. In the developing cortex, which is used as a model to study neuronal development, neurons born along the ventricles migrate outward, or radially, to form the layers of the cortical plate (Bystron et al. 2008). Similar to newly plated cultured neurons, newly born neurons in vivo initially have multiple (seemingly) equivalent processes, but prior to migrating, the neurons adopt a bipolar morphology, extending one process in the direction the neuron is traveling (so-called "leading process") and trailing a second process behind the cell body. The trailing process forms the axon, which grows as the neuron migrates. After the neuron reaches its terminal layer and ceases to migrate, the dendrites develop from the leading process. Unlike cultured neurons, however, which are typically plated on a uniform, growth-permissive substrate, newborn neurons in the developing brain likely respond to extracellular signals that influence which process becomes the axon. Neuronal polarization in vitro is generally thought to rely predominantly on intrinsic polarization machinery, whereas neuronal polarization in vivo likely results from an integration of internal and external signals.

\section{Cytoskeletal changes during neuronal polarization}

The question of how a single neuronal process is selected to become an axon was initially approached in cultured neurons by analyzing the role of the cytoskeleton in growing neurites. At the tip of each growing neurite is a specialized structure called the growth cone, which is comprised of a dynamic actin meshwork surrounding a relatively stable core of microtubules (MTs). The nascent axon usually has a slightly enlarged growth cone and grows more rapidly than the other neurites, raising the question of what cytoskeletal changes correspond with, and potentially lead to, axon specification. Destabilizing actin increases neurite growth (Forscher and Smith 1988) and adding cytochalasin D, a drug that inhibits actin polymerization, to the culture media of stage 2 neurons results in neurons forming multiple axons (Bradke and Dotti 1999). Futhermore, applying cytochalasin D to a single neurite causes the neurite to extend rapidly and become an axon. These data led to the hypothesis that at the tip of the future axon a loose actin meshwork allows MTs to extend into the growth cone to form a stable MT core that promotes axon growth (Forscher and Smith 1988; Bradke and Dotti 1999). Consistent with this model, stabilizing MTs by applying the drug taxol, or overexpressing CRMP-2, a molecule that promotes MT assembly, results in neurons with multiple axons (Inagaki et al. 2001; Fukata et al. 2002; Witte et al. 2008). In addition to changes in the actin and MT cytoskeletons, MT-associated proteins (MAPs) have also been implicated in neuronal polarity. The MAP Tau can influence MT dynamics and the movement of MT motors, and phosphorylation of Ser 262 causes Tau to dissociate from MTs. Dephosphorylated Tau is enriched in axons, whereas Tau phosphorylated at Ser 262 is found in dendrites. As described below, one way in which SAD is hypothesized to function during neuronal polarization is by affecting Tau phosphorylation.

Intrinsic signaling cascades: Akt promotes axon formation in vitro

Neurons are among many different types of cells that are polarized, and some of the first signaling molecules shown to regulate neuronal polarity in vitro were components of the conserved Par complex, which had been implicated previously in regulating the polarity of different cell types in multiple organisms (Goldstein and Macara 2007). Par3 and Par6, which together with aPKC comprise the Par complex, were initially identified as regulators of anterior-posterior polarity in the single cell worm embryo. In polarizing neurons, Par3 is localized to the nascent axon, and inhibiting Par complex activity blocks axon specification (Shi et al. 2003). The localization of Par3 to the axonal growth cone depends on PI3K, which acts upstream of the Par complex in polarizing neurons. PI3K signaling activates Akt (Shi et al. 2003), a kinase that promotes axon specification by inactivating GSK3 $\beta$. GSK3 $\beta$, also a kinase, restricts axon formation by inhibiting factors that promote axon growth, such as CRMP-2 and APC (Shi et al. 2004; Zhou et al. 2004; Jiang et al. 2005). PI3K also activates Akt in other cellular contexts where Akt targets molecules other than GSK3 $\beta$, raising the question of whether Akt might act upstream of other factors beside GSK3 $\beta$ in developing neurons. Indeed, the hypothesis that Akt inactivates Tsc1 and Tsc2 in polarizing neurons is the premise of the work of Choi et al. (2008) Some evidence hinting that Akt may have a target(s) other than GSK3 $\beta$ is that Myr-Akt increases both the number of axons and the total number of neu- 
ronal processes a neuron has, whereas the loss of GSK3 $\beta$ causes ectopic axons to form at the expense of dendrites (Jiang et al. 2005). Furthermore, a GSK3 $\beta$ mutant that cannot be phosphorylated by Akt has no effect on neuronal polarization (McManus et al. 2005; Gartner et al. 2006). The work of Choi et al. (2008) reveals that Akt targets and blocks the activity of the Tsc1-Tsc2 complex, which, similar to GSK3 $\beta$, antagonizes axon formation. Although it is not clear whether the Tsc1-Tsc2 complex also influences the overall number of processes a neuron extends, these experiments indicate that the Tsc1-Tsc 2 complex is a target of Akt in polarizing neurons.

SAD kinases are required for neuronal polarity in vitro and in vivo

The differential localization of proteins and organelles to axons or dendrites underlies the functional polarity of neurons. In Caenorhabditis elegans, the kinase SAD-1 localizes to synaptic sites along the axon and is necessary for proper presynaptic vesicle distribution at synapses (Crump et al. 2001). Overexpressing $S A D-1$ causes presynaptic proteins and vesicles to localize ectopically to dendrites, suggesting that SAD-1 might regulate some aspects of neuronal polarity. The two mammalian $S A D-1$ homologs, $S A D-A$ and $S A D-B$, are expressed predominantly in the developing murine nervous system (Kishi et al. 2005). In the brains of $S A D-A, S A D-B$ double knockout ( $S A D$-null) mice, cortical plate layering is abnormal and major axonal tracts are missing. Closer examination revealed that $S A D$-null neurons did not have clear axons or dendrites, and the neurites frequently extended tangentially, rather than radially as in wild type. Strikingly, the processes of hippocampal neurons cultured from $S A D$-null brains exhibited mixed axon and dendritic characteristics: Each process contained both MAP2, which is normally enriched in dendrites, and high levels of dephosphorylated Tau (recognized by the anti-Tau-1 antibody), which is typically a characteristic of axons. Unlike control neurons, which have long axons and relatively short dendrites, the $S A D$-null neurons had processes of equivalent lengths, and the average process length was intermediate between the normal length of an axon or dendrite. How might SAD influence axon and dendrite formation? The similarity of the SAD kinase domain to PAR-1 and MARK suggested SAD might phosphorylate MAPs. As described above, the binding of Tau to MTs is regulated by phosphorylation of Ser 262; dephosphorylated Tau is concentrated in axons and Tau phosphorylated at Ser 262 is enriched in dendrites. Overexpressing $S A D-A$ or $S A D-B$ increased the fraction of Tau phosphorylated at Ser 262 without affecting overall Tau levels, indicating that SAD promotes the phosphorylation of Tau at Ser 262. Consistent with these in vitro results, in $S A D$-null brains dephosphorylated Tau is present not only in the axon-rich intermediate zone, but also the cortical plate, where dendrites elaborate.

\section{LKB1 activates SAD kinases in polarizing neurons}

SAD is necessary for axons and dendrites to develop their distinct properties, yet SAD-A and SAD-B are found in both axons and dendrites, raising several questions, including: What is upstream of SAD, and is SAD differentially active in axons versus dendrites? One candidate SAD regulator is the PAR-4 ortholog LKB1, which in conjunction with STRAD and MO25 phosphorylates and activates AMPK and AMPK-related kinases such as PAR-1 (Spicer and Ashworth 2004). Similar to knocking out $S A D-A$ and $S A D-B$, eliminating or reducing LKB1 function in the developing mouse brain results in neurons that lack axons (Barnes et al. 2007; Shelly et al. 2007). Moreover, cultured neurons lacking LKB1 developed neurites of equivalent length that contained both dephosphorylated Tau and MAP2, indicating that LKB1, like SAD, is necessary for processes to be specified as either axons or dendrites. Biochemical experiments revealed that LKB1 phosphorylates SAD-A and SAD-B, and phosphorylated SAD-A and SAD-B are greatly reduced in the cortices of $L K B 1$ knockout mice (Barnes et al. 2007). Significantly, Western blot analysis showed that LKB1mediated SAD phosphorylation correlates with the phosphorylation of Tau at Ser 262, indicating that LKB1 is necessary for SAD activity. Where in neurons is SAD active? Both phosphorylated LKB1 and phosphorylated SAD are concentrated in axons, indicating that LKB1 activates SAD preferentially within axons, and suggesting that LKB1 and SAD function to specify the axon. Consistently, increasing LKB1 activity results in neurons with multiple axons (Barnes et al. 2007; Shelly et al. 2007). Although it seems somewhat contradictory that SAD-A and SAD-B are active in the axon, which typically has low levels of phosphorylated Tau, the KXGS motif in Tau that SAD kinase recognizes is shared by other proteins, including other MAPs, making it likely that SAD regulates additional targets in polarizing neurons.

\section{A role for local translation in neuronal polarity and axon growth}

By implicating TORC1 in neuronal polarity, Choi et al. (2008) raise the possibility that local translation is critical to axon formation. Local translation has been previously shown to be crucial for establishing anteriorposterior polarity in oocytes in the fruit fly germ line, and different mRNAs are asymmetric localized within both neural precursors and mature neurons, all of which indicate that local protein synthesis is necessary for multiple aspects of cell and neuronal polarity. Furthermore, work with cultured mammalian neurons has revealed that axon growth and guidance depends on local translation within growth cones (Lin and Holt 2007). In retinal axon growth cones the chemoattractant Netrin-1 acts upstream of Tor to promote protein synthesis in response to receptor activity (Campbell and Holt 2001; Leung et al. 2006). Netrin-1 activity induces a gradient of Tor activity across the growth cone, thereby creating lo- 
cal differences in protein levels that cause one side of the growth cone to extend relative to the other, enabling the axon to turn. A link between neuronal polarity and chemoattractant guidance molecules such as Netrin-1 was originally suggested by the observation that the axon of polarizing neurons always emerges from the side of the cell body closest to the pathway it will follow. This idea that extracellular signaling molecules orient the polarity of neurons relative to their surroundings has been supported by studies in C. elegans showing that the attractive guidance cues Netrin-1 and Wnt are necessary for axons to extend in their proper direction (Adler et al. 2006; Hilliard and Bargmann 2006; Prasad and Clark 2006). Although it is not known what extracellular signaling molecules regulate the activity of TORC1 in polarizing neurons, Choi et al. (2008) report that TORC1 activity is high in axons and that TORC1 is crucial for axon formation, two pieces of data that suggest local translation has a role in distinguishing the nascent axon from other processes.

\section{The Tsc1-Tsc2 complex and TORC1 regulate axon formation and modulate SAD protein levels}

Cortical tubers that occur in TSC patients likely result from abnormal cortical development. Whereas prior studies focused on the roles of Tsc1 and Tsc2 in mature neurons, Choi et al. (2008) examined the effect of decreased Tsc1-Tsc2 complex activity during earlier stages of neurodevelopment. The investigators started with the hypothesis that Akt might regulate Tsc2 activity not only in growing cells, but also in polarizing neurons. Akt is active in axons, and consistently, the amount of phosphorylated, inactive Tsc2 is higher in axons than dendrites. This suggests that the Tsc1-Tsc2 complex antagonizes axon formation, and, consistently, reducing Tsc2 levels in cultured hippocampal neurons results in neurons with multiple axons. In Tsc1 mutant cortices, axonal markers stained not only the intermediate zone, where axons normally grow, but also other cortical layers, including the cortical plate; this phenotype is opposite to that due to the loss of SAD or LKB1, which inhibit axon formation and result in a decrease in axonal marker staining. Although LKB1 positively regulates Tsc1-Tsc2 complex activity in other contexts, it is unlikely to activate the Tsc1-Tsc2 complex in neurons. LKB1 activates the Tsc1-Tsc2 complex via AMPK, and neither the amount of AMPK nor its phosphorylation state is changed in LKB1-null brains (Barnes et al. 2007), indicating that LKB1 does not regulate AMPK in the nervous system. In addition to affecting the morphology of mature neurons, the loss of Tsc1 perturbs the morphology of migrating neurons such that many of the neurons extend their processes laterally, rather than apically. This defect in migrating neurons foreshadows morphological abnormalities in mature neurons, as it has been reported that apical dendrites, which develop from the leading (or apical) process, are frequently misoriented in Tsc1 mutant cortices (Meikle et al. 2008).

How does the Tsc1-Tsc2 complex regulate axon for- mation? First, Choi et al. (2008) provide evidence that, as in other scenarios, the Tsc1-Tsc2 complex regulates TORC1 activity: Axons have elevated levels of phosphorylated $56 \mathrm{~K}$, which indicates increased TORC1 activity, and rapamycin suppresses the formation of multiple axons in Tsc2 mutant neurons. As TORC1 regulates protein translation, the investigators next looked for changes in the level of proteins previously implicated in neuronal polarity, including SAD-A and SAD-B. Western blot analysis revealed that the amount of SAD-A and SAD-B is increased in Tsc2 mutant neurons. There is also an increase in SAD phosphorylation as well as the phosphorylation of Tau at Ser 262, suggesting that the increase in SAD protein levels correlates with an increase in SAD activity. Additionally, treating the Tsc2 mutant neurons with rapamycin reduces SAD-A levels to normal. However, rapamycin treatment does not reduce SAD-A protein below normal levels, suggesting that although TORC1 can elevate the amount of SAD protein, TORC1 is not necessary for the basal translation of SAD. This fits with the observations that SAD kinases are present in both axons and dendrites, but that TORC1 is active specifically in axons. Yet, it is likely that the Tsc1-Tsc2 complex and TORC1 have targets other than SAD, since knocking down SAD-A and SAD-B in cultured neurons with low levels of Tsc2 only partially suppresses the multiaxon phenotype. The work of Choi et al. (2008) suggests that the Tsc1-Tsc2 complex holds in check the local translation of $S A D$ and possibly other mRNAs that promote axon formation, thus allowing for a temporally and spatially controlled response to a polarizing cue(s). In the future, it will be of interest to determine what signaling molecule(s) act upstream of Akt and the Tsc1-Tsc2 complex. One possible candidate is BDNF, which in growing cells regulates Akt and Tsc1Tsc2 complex function (Swiech et al. 2008), and in cultured neurons promotes axon specification by increasing LKB1 activity (Shelly et al. 2007). This raises the possibility that a single signaling molecule may be atop two pathways, one of which (LKB1/SAD) impinges on the MT cytoskeleton, while the other (Akt/Tsc1-Tsc2 complex/TORC1) regulates local protein synthesis.

\section{Implications for TSC pathogenesis}

Choi et al. (2008) show that SAD-A levels are often notably increased in the giant cells of a cortical tuber resected from a TSC patient. This is a significant finding, suggesting a correlation between elevated amounts of SAD-A and cortical tuber formation. However, it is not clear why SAD levels differ between the giant cells and surrounding neurons in the tuber. Whereas harmatomas in organs such as the kidney often display loss of heterozygosity (LOH) consistent with the tumor suppressor model, LOH in cortical tubers is rare (Henske et al. 1996; Niida et al. 2001). This raises the question of whether the loss of one copy of Tsc1 or Tsc2 is sufficient to significantly increase the amount of SAD and affect neuronal polarity and/or morphology. LOH in cortical tubers is an important issue to address given the debate about 
whether lesion load correlates with the severity of neurological deficits, particularly in light of recent work that indicates that working memory deficits correlate with bilaterally symmetric abnormalities in the gray and white matter (Ridler et al. 2007).

\section{References}

Adler, C.E., Fetter, R.D., and Bargmann, C.I. 2006. UNC-6/Netrin induces neuronal asymmetry and defines the site of axon formation. Nat. Neurosci. 9: 511-518.

Barnes, A.P., Lilley, B.N., Pan, Y.A., Plummer, L.J., Powell, A.W., Raines, A.N., Sanes, J.R., and Polleux, F. 2007. LKB1 and SAD kinases define a pathway required for the polarization of cortical neurons. Cell 129: 549-563.

Bradke, F. and Dotti, C.G. 1999. The role of local actin instability in axon formation. Science 283: 1931-1934.

Bystron, I., Blakemore, C., and Rakic, P. 2008. Development of the human cerebral cortex: Boulder Committee revisited. Nat. Rev. Neurosci. 9: 110-122.

Campbell, D.S. and Holt, C.E. 2001. Chemotropic responses of retinal growth cones mediated by rapid local protein synthesis and degradation. Neuron 32: 1013-1026.

Choi, Y.-J., Di Nardo, A., Kramvis, I., Meikle, L., Kwiatkowski, D.J., Sahin, M., and He, X. 2008. Tuberous sclerosis complex proteins control axon formation. Genes \& Dev. (this issue). doi: $10.1101 / \mathrm{gad} .1685008$

Crino, P.B. 2004. Molecular pathogenesis of tuber formation in tuberous sclerosis complex. J. Child Neurol. 19: 716-725.

Crino, P.B., Nathanson, K.L., and Henske, E.P. 2006. The tuberous sclerosis complex. N. Engl. J. Med. 355: 1345-1356.

Crump, J.G., Zhen, M., Jin, Y., and Bargmann, C.I. 2001. The SAD-1 kinase regulates presynaptic vesicle clustering and axon termination. Neuron 29: 115-129.

Dotti, C.G., Sullivan, C.A., and Banker, G.A. 1988. The establishment of polarity by hippocampal neurons in culture. $J$. Neurosci. 8: 1454-1468.

Ehninger, D., Han, S., Shilyansky, C., Zhou, Y., Li, W., Kwiatkowski, D.J., Ramesh, V., and Silva, A.J. 2008. Reversal of learning deficits in a $\mathrm{Tsc}^{+/-}$mouse model of tuberous sclerosis. Nat. Med. 14: 843-848.

Forscher, P. and Smith, S.J. 1988. Actions of cytochalasins on the organization of actin filaments and microtubules in a neuronal growth cone. I. Cell Biol. 107: 1505-1516.

Fukata, Y., Itoh, T.J., Kimura, T., Ménager, C., Nishimura, T., Shiromizu, T., Watanabe, H., Inagaki, N., Iwamatsu, A., Hotani, H., et al. 2002. CRMP-2 binds to tubulin heterodimers to promote microtubule assembly. Nat. Cell Biol. 4: 583591.

Gao, X. and Pan, D. 2001. TSC1 and TSC2 tumor suppressors antagonize insulin signaling in cell growth. Genes \& Dev. 15: $1383-1392$.

Gartner, A., Huang, X., and Hall, A. 2006. Neuronal polarity is regulated by glycogen synthase kinase-3 (GSK-3 $\beta$ ) independently of Akt/PKB serine phosphorylation. J. Cell Sci. 119: 3927-3934.

Goldstein, B. and Macara, I.G. 2007. The PAR proteins: Fundamental players in animal cell polarization. Dev. Cell 13: 609-622.

Halpain, S., Spencer, K., and Graber, S. 2005. Dynamics and pathology of dendritic spines. Prog. Brain Res. 147: 29-37.

Henske, E.P., Scheithauer, B.W., Short, M.P., Wollmann, R., Nahmias, J., Hornigold, N., van Slegtenhorst, M., Welsh, C.T., and Kwiatkowski, D.J. 1996. Allelic loss is frequent in tuberous sclerosis kidney lesions but rare in brain lesions.
Am. J. Hum. Genet. 59: 400-406.

Hilliard, M.A. and Bargmann, C.I. 2006. Wnt signals and frizzled activity orient anterior-posterior axon outgrowth in C. elegans. Dev. Cell 10: 379-390.

Huang, J. and Manning, B.D. 2008. The TSC1-TSC2 complex: A molecular switchboard controlling cell growth. Biochem. J. 412: 179-190.

Inagaki, N., Chihara, K., Arimura, N., Ménager, C., Kawano, Y., Matsuo, N., Nishimura, T., Amano, M., and Kaibuchi, K. 2001. CRMP-2 induces axons in cultured hippocampal neurons. Nat. Neurosci. 4: 781-782.

Jaworski, J. and Sheng, M. 2006. The growing role of mTOR in neuronal development and plasticity. Mol. Neurobiol. 34: 205-219.

Jiang, H., Guo, W., Liang, X., and Rao, Y. 2005. Both the establishment and the maintenance of neuronal polarity require active mechanisms: Critical roles of GSK- $3 \beta$ and its upstream regulators. Cell 120: 123-135.

Kishi, M., Pan, Y.A., Crump, J.G., and Sanes, J.R. 2005. Mammalian SAD kinases are required for neuronal polarization. Science 307: 929-932.

Leung, K.M., van Horck, F.P., Lin, A.C., Allison, R., Standart, N., and Holt, C.E. 2006. Asymmetrical $\beta$-actin mRNA translation in growth cones mediates attractive turning to netrin1. Nat. Neurosci. 9: 1247-1256.

Lin, A.C. and Holt, C.E. 2007. Local translation and directional steering in axons. EMBO J. 26: 3729-3736.

McManus, E.J., Sakamoto, K., Armit, L.J., Ronaldson, L., Shpiro, N., Marquez, R., and Alessi, D.R. 2005. Role that phosphorylation of GSK3 plays in insulin and Wnt signalling defined by knockin analysis. EMBO J. 24: 1571-1583.

Meikle, L., Talos, D.M., Onda, H., Pollizzi, K., Rotenberg, A., Sahin, M., Jensen, F.E., and Kwiatkowski, D.J. 2007. A mouse model of tuberous sclerosis: Neuronal loss of Tsc1 causes dysplastic and ectopic neurons, reduced myelination seizure activity, and limited survival. J. Neurosci. 27: 55465558.

Meikle, L., Pollizzi, K., Egnor, A., Kramvis, I., Lane, H., Sahin, M., and Kwiatkowski, D.J. 2008. Response of a neuronal model of tuberous sclerosis to mammalian target of rapamycin (mTOR) inhibitors: Effects on mTORCl and Akt signaling lead to improved survival and function. J. Neurosci. 28: 5422-5432.

Niida, Y., Stemmer-Rachamimov, A.O., Logrip, M., Tapon, D., Perez, R., Kwiatkowski, D.J., Sims, K., MacCollin, M., Louis, D.N., and Ramesh, V. 2001. Survey of somatic mutations in tuberous sclerosis complex (TSC) hamartomas suggests different genetic mechanisms for pathogenesis of TSC lesions. Am. J. Hum. Genet. 69: 493-503.

Potter, C.J., Huang, H., and Xu, T. 2001. Drosophila Tsc1 functions with Tsc2 to antagonize insulin signaling in regulating cell growth, cell proliferation, and organ size. Cell 105: 357368 .

Prasad, B.C. and Clark, S.G. 2006. Wnt signaling establishes anteroposterior neuronal polarity and requires retromer in C. elegans. Development 133: 1757-1766.

Ridler, K., Suckling, J., Higgins, N.J., de Vries, P.J., Stephenson, C.M., Bolton, P.F., and Bullmore, E.T. 2007. Neuroanatomical correlates of memory deficits in tuberous sclerosis complex. Cereb. Cortex 17: 261-271.

Shelly, M., Cancedda, L., Heilshorn, S., Sumbre, G., and Poo, M.M. 2007. LKB1/STRAD promotes axon initiation during neuronal polarization. Cell 129: 565-577.

Shi, S.H., Jan, L.Y., and Jan, Y.N. 2003. Hippocampal neuronal polarity specified by spatially localized $\mathrm{mPar} 3 / \mathrm{mPar} 6$ and PI 3-kinase activity. Cell 112: 63-75. 
Shi, S.H., Cheng, T., Jan, L.Y., and Jan, Y.N. 2004. APC and GSK- $3 \beta$ are involved in mPar3 targeting to the nascent axon and establishment of neuronal polarity. Curr. Biol. 14: 20252032.

Spicer, J. and Ashworth, A. 2004. LKB1 kinase: Master and commander of metabolism and polarity. Curr. Biol. 14: R383R385. doi: 10.1016/j.cub.2004.05.012.

Swiech, L., Perycz, M., Malik, A., and Jaworski, J. 2008. Role of mTOR in physiology and pathology of the nervous system. Biochim. Biophys. Acta 1784: 116-132.

Tapon, N., Ito, N., Dickson, B.J., Treisman, J.E., and Hariharan, I.K. 2001. The Drosophila tuberous sclerosis complex gene homologs restrict cell growth and cell proliferation. Cell 105: 345-355.

Tavazoie, S.F., Alvarez, V.A., Ridenour, D.A., Kwiatkowski, D.J., and Sabatini, B.L. 2005. Regulation of neuronal morphology and function by the tumor suppressors Tscl and Tsc2. Nat. Neurosci. 8: 1727-1734.

Uhlmann, E.J., Wong, M., Baldwin, R.L., Bajenaru, M.L., Onda, H., Kwiatkowski, D.J., Yamada, K., and Gutmann, D.H. 2002. Astrocyte-specific TSC1 conditional knockout mice exhibit abnormal neuronal organization and seizures. Ann. Neurol. 52: 285-296.

Witte, H., Neukirchen, D., and Bradke, F. 2008. Microtubule stabilization specifies initial neuronal polarization. J. Cell Biol. 180: 619-632.

Zhou, F.Q., Zhou, J., Dedhar, S., Wu, Y.H., and Snider, W.D. 2004. NGF-induced axon growth is mediated by localized inactivation of GSK-3 $\beta$ and functions of the microtubule plus end binding protein APC. Neuron 42: 897-912. 


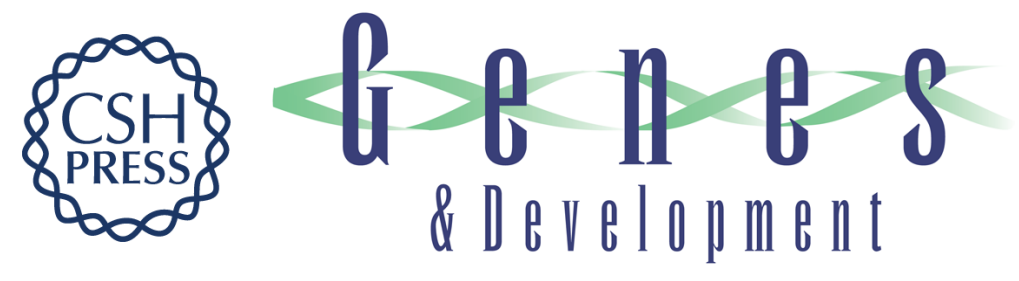

\section{The Tsc1-Tsc2 complex influences neuronal polarity by modulating TORC1 activity and SAD levels}

Jill Wildonger, Lily Yeh Jan and Yuh Nung Jan

Genes Dev. 2008, 22:

Access the most recent version at doi:10.1101/gad.1724108

\section{Related Content Tuberous sclerosis complex proteins control axon formation \\ Yong-Jin Choi, Alessia Di Nardo, loannis Kramvis, et al. \\ Genes Dev. September , 2008 22: 2485-2495 \\ References This article cites 42 articles, 12 of which can be accessed free at: \\ http://genesdev.cshlp.org/content/22/18/2447.full.html\#ref-list-1 \\ Articles cited in: \\ http://genesdev.cshlp.org/content/22/18/2447.full.html\#related-urls \\ License
Email Alerting Receive free email alerts when new articles cite this article - sign up in the box at the top Service right corner of the article or click here.

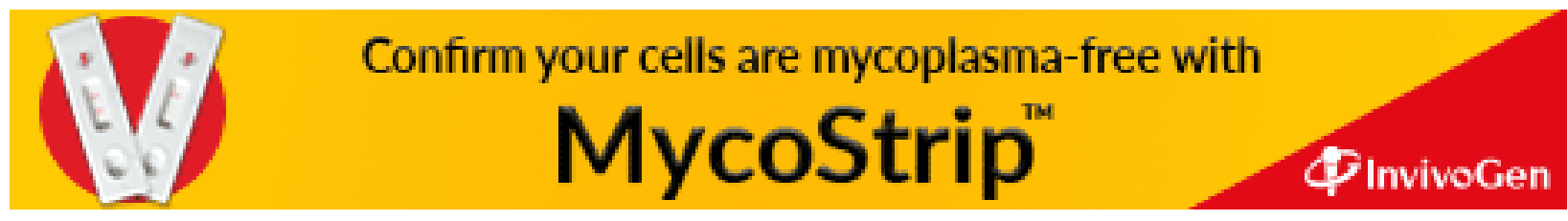

